\title{
NONLINEAR OBSERVER BASED SENSORLESS DIRECT TORQUE CONTROL OF INDUCTION MOTOR
}

\author{
Dinesh Pai A, Purnaprajna R Mangsuli, N J Rao \\ Centre for Electronics Design and Technology \\ Indian Institute of Science, Bangalore -560 012, India \\ E - mail: adpai@ cedt.iisc.ernet.in
}

\begin{abstract}
Induction motor speed control is an area of research that has been in prominence for some time now. Recent advances in this field have made it possible to replace the DC motor by induction machines, even in applications that demand a fast dynamic response. Many industrial applications demand speed sensorless operations, due to various reasons. It is also required to strictly maintain the speed of the motor within certain permissible tolerance, irrespective of the load changes that occur in the system. Unless prior knowledge of the load characteristics is known, it is very difficult to compensate for the same. Direct Torque Control (DTC) of induction motor is a popular method because of the resulting fast dynamic response of the motor, lower sensitivity to motor parameter variations and relatively low switching harmonics in the inverter. However, the present DTC approach is unsuitable for highperformance applications because of the need of a speed sensor for increased accuracy, the absence of any error decay mechanism, and the requirement of prior knowledge of the load or disturbance characteristics. In this paper, a nonlinear observer is designed for the stator flux, speed, and load torque estimation that will take care of the above limitations. The estimated values along with other measured states are used for the closed loop speed sensorless control operation of the induction motor. Simulations are done and the results discussed.
\end{abstract}

Keywords: Motor control, Nonlinear observer, State convergence

Symbols:

$\alpha, \beta$ - stationary reference coordinates

$V_{s \alpha}, V_{s \beta}-$ stator voltage in $\alpha-\beta$ coordinates

$I_{s \alpha}, I_{s \beta}$ - stator current in $\alpha-\beta$ coordinates

$\varphi_{s \alpha}, \varphi_{s \beta}$ - stator flux in $\alpha-\beta$ coordinates

$\omega_{r}$ - motor shaft speed

$\delta$ - torque angle

$x, u, y$ - system states, input, output

$T_{L}$ - load torque

$Q$ - observability matrix

$\phi$ - transformation matrix

$a_{m}$ - Lipschitz constant

$V$ - Lyapunov function

\section{Introduction}

Much effort has been put on the development of highperformance sensorless speed control of induction motor drives for the last few years. A radical step in induction motor control strategies was the development of Direct
Torque Control method [1,2,3,4]. This stator flux orientated scheme is based on the limit cycle control of both flux and torque. In principle, it uses a switching table for selecting the inverter output voltage vector based on the instantaneous requirement of torque and flux, to get fast torque response and low inverter switching frequency.

For high-performance induction motor drives, information regarding the stator flux, motor speed, and load torque characteristics are to be given to the controller. To reduce the total hardware complexity, space and cost, to increase the mechanical robustness and reliability of the drive, and to obtain increased noise immunity, it is desirable to eliminate sensors, particularly for speed and position measurements. An electromechanical sensor increases the system inertia, which is undesirable in high-performance drives. Moreover, the load torque characteristics may not be known in advance in most of the cases, which makes it difficult to strictly maintain the motor speed within the specification. Conventional open loop estimation of stator flux from the monitored voltages and currents is not accurate enough especially at very low speeds owing to the considerable voltage drop across the stator resistance and due to the absence of error decay mechanism [5]. This work is oriented towards the improvement to the existing Direct Torque Control methods for induction motor, with an emphasis on highperformance speed sensorless operation under changes in load conditions. A nonlinear observer based on advanced nonlinear control theory is designed for the stator flux, speed, and load torque estimation. These estimated values along with other measured states are used for the closed loop speed control of the induction motor.

\section{Principle of Direct Torque Control}

The expression for the developed torque of an induction motor is given by (1).

$$
\begin{aligned}
& T=\frac{N_{p} \cdot M}{\sigma \cdot L_{s} \cdot L_{r}} \cdot \Phi_{s} \cdot \Phi_{r} \cdot \sin \delta \\
& \text { where, } \sigma=1-\frac{M^{2}}{L_{s} \cdot L_{r}} .
\end{aligned}
$$

Under normal operating conditions, the amplitude of the working flux is kept constant at the maximum value for maximum utilization of magnetic material. Hence the developed torque is proportional to the sine of the torque angle ' $\delta$ ', i.e., the angle between stator and rotor fluxes, and can be controlled by suitably changing the torque angle. Since the time constant of rotor current is normally large compared to stator, the stator flux is 
accelerated or decelerated with respect to the rotor flux to change the torque angle.

The voltage source inverter can be modeled as shown in fig. 1, where $S_{a}, S_{b}, S_{c}$ are the switching states. Eight output voltage vectors $V_{0}$ to $V_{7}\{000,100,110,010,011$, $001,101,111\}$ as shown in fig. 2 are obtained for different switch combinations. Out of these $V_{0}$ and $V_{7}$ are zero voltage vectors. Inverter output voltage is given by the expression (2), where $\mathrm{V}_{\mathrm{dc}}$ is the DC - link voltage.

$$
V_{s}=\sqrt{\frac{2}{3}} \cdot V_{d c} \cdot\left(S_{a}+S_{b} \cdot e^{j 2 \pi / 3}+S_{c} \cdot e^{-j 2 \pi / 3}\right)
$$

Assuming the voltage drop across the stator resistance to be small, stator flux variation can be expressed as (3).

$$
\begin{aligned}
& \varphi_{s}=\int_{0}^{t}\left[V_{s}-I_{s}, R_{s}\right] d t \\
& \Delta \varphi_{s} \cong V_{s} \Delta t
\end{aligned}
$$

This indicates that ' $\varphi_{s}$ ' moves on a locus, with constant velocity determined by the selected voltage vector and the duration ' $\Delta t$ ' for which it is applied. The output voltage vectors among $\mathrm{V}_{0}-\mathrm{V}_{7}$ are selected to change the torque angle. This is done based on the instantaneous torque requirement, ensuring the error between $\left|\varphi_{s}\right|$ and $\left|\varphi_{s}\right|^{*}$ (reference) to be within a tolerance band. It can be seen that the selection of voltage vector also depends on the direction of $\varphi_{s}$. As shown in fig. 2 , the voltage yector changes periodically in steps of $\pi / 3$ radian. Accordingly to discriminate the direction, $\alpha-\beta$ plane is divided into six sectors $\theta(\mathbf{k})$, $k=1,2,3,4,5,6$. It can be seen from fig. 3 , that in any sector ' $k$ ', for counter clockwise rotation, voltage vectors $\mathrm{V}(\mathrm{k}+1)$ and $\mathrm{V}(\mathrm{k}+2)$ accelerates the stator flux, while $\mathrm{V}(\mathrm{k}-1)$ and $\mathrm{V}(\mathrm{k}-2)$ decelerates. Similarly $\mathrm{V}(\mathrm{k}), \mathrm{V}(\mathrm{k}+1)$ and $V(k-1)$ increases the amplitude of stator flux while $\mathrm{V}(\mathrm{k}+2), \mathrm{V}(\mathrm{k}+3)$ and $\mathrm{V}(\mathrm{k}-2)$ decreases. For clockwise rotation, reverse happens. The zero voltage vectors does not affect the stator flux substantially, with the exception of the small flux weakening due to the voltage drop across the stator resistance. Inverter output voltage vectors are selected based on the sector in which the flux lies at the instant and the instantaneous requirements of the torque and the stator flux. Two- and three-level hysterisis comparator digitizes the flux and torque errors, respectively. The digitized flux direction is determined by comparing the $\alpha-\beta$ components of the flux linkage vector with its amplitude. These digital signals, i.e., one bit of flux, two bits of torque, and three bits of sector refer the optimum switching table. Table -1 summarizes the combined action of each voltage space vector on the stator flux and the torque. As it appears from the table, for both positive and negative motor speeds, an increment of torque $(\uparrow)$ is obtained by two vectors only,

Table - 1. Effect of voltages on flux and torque

\begin{tabular}{|c|c|c|c|c|c|c|c|}
\hline & $\begin{array}{c}\mathrm{V} \\
(\mathrm{k}-2)\end{array}$ & $\begin{array}{c}\mathrm{V} \\
(\mathrm{k}-1)\end{array}$ & $\begin{array}{c}\mathrm{v} \\
(\mathrm{k})\end{array}$ & $\begin{array}{c}\mathrm{v} \\
(\mathrm{k}+1)\end{array}$ & $\begin{array}{c}\mathrm{V} \\
(\mathrm{k}+2)\end{array}$ & $\begin{array}{c}\mathrm{V} \\
(\mathrm{k}+3)\end{array}$ & $\begin{array}{c}\mathrm{V}_{0} \\
\mathrm{or} \\
\mathrm{V}_{7}\end{array}$ \\
\hline$\varphi_{\mathrm{s}}$ & $\downarrow$ & $\uparrow$ & $\uparrow \uparrow$ & $\uparrow$ & $\downarrow$ & $\downarrow \downarrow$ & $\uparrow \downarrow$ \\
\hline $\begin{array}{c}\mathrm{T} \\
\omega_{3}>0\end{array}$ & $\downarrow \downarrow$ & $\downarrow \downarrow$ & $\downarrow$ & $\uparrow$ & $\uparrow$ & $\downarrow$ & $\downarrow$ \\
\hline $\begin{array}{c}\mathrm{T} \\
\omega_{3}<0\end{array}$ & $\downarrow$ & $\downarrow$ & $\uparrow$ & $\uparrow \uparrow$ & $\uparrow \uparrow$ & $\uparrow$ & $\uparrow$ \\
\hline
\end{tabular}

Table - 2. Switching strategies

\begin{tabular}{|c|c|c|c|c|}
\hline & $\mathbf{T} \Uparrow, \varphi_{2} \Uparrow$ & $\mathbf{T} \Uparrow, \boldsymbol{\varphi}_{3} \Downarrow$ & $\mathbf{T} \Downarrow, \boldsymbol{\varphi}_{3} \Uparrow$ & $\mathbf{T} \mathbb{V}_{2} \boldsymbol{\varphi}_{3} \Downarrow$ \\
\hline Solution - A & $\mathrm{V}(\mathbf{k}+1)$ & $\mathrm{V}(\mathbf{k}+2)$ & $\mathrm{V}_{0}, \mathrm{~V}_{7}$ & $\mathrm{~V}_{0}, \mathrm{~V}_{7}$ \\
\hline Solution - B & $\mathrm{V}(\mathbf{k}+1)$ & $\mathrm{V}(\mathbf{k}+2)$ & $\mathrm{V}(\mathbf{k})$ & $\mathrm{V}_{0}, \mathrm{~V}_{7}$ \\
\hline Solution - C & $\mathrm{V}(\mathbf{k}+1)$ & $\mathrm{V}(\mathbf{k}+2)$ & $\mathrm{V}(\mathbf{k})$ & $\mathrm{V}(\mathbf{k}+3)$ \\
\hline Solution - D & $\mathrm{V}(\mathbf{k}+1)$ & $\mathrm{V}(\mathbf{k}+2)$ & $\mathrm{V}(\mathbf{k}-1)$ & $\mathrm{V}(\mathbf{k}-2)$ \\
\hline
\end{tabular}

that are $\mathrm{V}(\mathrm{k}+1)$ and $\mathrm{V}(\mathrm{k}+2)$. Conversely, a decrement of torque $(\downarrow)$ can be obtained by applying $\mathrm{V}(\mathrm{k}-1)$ or $\mathrm{V}(\mathrm{k}-2)$. The radial voltage space vectors act on the torque in accordance with the motor speed direction. In this table, a single arrow means a small influence on the flux or torque variations, whilst two arrows denote a larger influence.

The hysterisis band technique leads to four possible combinations with regard to the stator flux and torque errors. Each solution affects the drive behavior in terms of torque and current ripple, switching frequency and two- or four-quadrant operation capability. In Table -2 , four switching solutions are given. Strategies A, B and C can be used for two-quadrant operation, while strategy $D$ is suitable for four-quadrant operation.

At low speeds, it is better to select suitable active voltage vectors to reduce the torque angle rather than zero voltage vector to get fast torque response. A convenient control technique, which utilizes a different voltage space vector selection strategy, according to the operating speed range can be employed. The schematic of speed control of induction motor using Direct Torque Control method is shown in fig. 4.

\section{Flux Estimation and Associated Problems}

Performance of the system depends greatly on the accuracy with which the stator flux and speed are estimated and these in turn depend on the accuracy of the monitored voltages and currents. Errors may occur in the monitored voltages and currents due to the following factors: phase shift in the measured values (because of sensors), magnitude errors because of conversion factors and gain, offset in the measurement system, quantisation error in the digital system, sensors and measurement noises. Conventional methods use open loop estimation of stator flux (3) by simply integrating the stator voltage $\mathrm{V}_{s}$. For better accuracy, drop across the stator resistance is also to be considered. The main disadvantage of open loop estimation is that it is parameter sensitive and any mismatch in the initial conditions because of the reasons mentioned above, will adversely affect the system response both in transient and steady states. It may sometimes introduce steady state output bias and cause even system instability. Any sort of error introduced in the speed estimation also cannot be overcome and can lead to an increasing deviation of the result from the actual value in the absence of any error decay mechanism. In addition, open loop speed estimation requires differentiation, which is undesirable in view of the associated noise amplification. Compensation of the load torque is another difficult task that is to be tackled in high-performance drives. Closed-loop observers are better choices for improving the robustness against 
parameter mismatch and also signal to noise ratio. Since the induction motor is nonlinear in nature, linear observers may not offer required performance over the entire operating range. Alternatively nonlinear observers can be employed. Recent developments in nonlinear control theory lead to several well-established nonlinear observer theories. It has also been proved that the convergence of states to its true value is guaranteed, subject to satisfying certain stability conditions. We consider one such nonlinear observer for the highperformance induction motor speed sensorless control under changing load conditions, making use of the excellent features of DTC technique, compared to other conventional methods such as vector control.

\section{Induction Motor Model and Nonlinear Observer}

A nonlinear system can be represented as given in (4) wherein, $G$ and $h$ are considered to be functions of system states.

$$
\left.\begin{array}{l}
\dot{x}=f(x)+G(x) \cdot u \\
y=h(x)
\end{array}\right\}
$$

As stator flux is controlled directly, stator flux oriented model of the induction motor in $\alpha-\beta$ coordinates (stator fixed reference frame) is considered. Since the load dynamics is much slower compared to the system dynamics, load dynamics can be considered as $\dot{T}_{L}=0$. This can be augmented with the motor model.

Defining states ' $x$ ', input ' $u$ ', output ' $y$ ' as in (5), the augmented induction motor can be modelled as (6).

$$
\begin{aligned}
& x=\left[\begin{array}{llllll}
x_{1} & x_{2} & x_{3} & x_{4} & x_{5} & x_{6}
\end{array}\right]^{T} \\
& =\left[\begin{array}{llllll}
I_{s \alpha} & I_{s \beta} & \varphi_{s \alpha} & \varphi_{s \beta} & \omega_{r} & T_{L}
\end{array}\right]^{T} \\
& u=\left[\begin{array}{ll}
u_{1} & u_{2}
\end{array}\right]^{T}=\left[\begin{array}{ll}
V_{s \alpha} & V_{s \beta}
\end{array}\right]^{T} \\
& y=\left[\begin{array}{ll}
y_{1} & y_{2}
\end{array}\right]^{T}=\left[\begin{array}{ll}
I_{s \alpha} & I_{s \beta}
\end{array}\right]^{T} \\
& \dot{x}_{1}=F_{11} \cdot x_{1}+F_{12} \cdot x_{2} \cdot x_{5}+F_{13} \cdot x_{3}+F_{14} \cdot x_{4} \cdot x_{5}+g_{11} \cdot u_{1} \\
& =f_{1}(x)+g_{11} u_{1} \\
& \dot{x}_{2}=F_{21} \cdot x_{1} \cdot x_{5}+F_{22} \cdot x_{2}+F_{23} \cdot x_{3} \cdot x_{5}+F_{24} \cdot x_{4}+g_{22} \cdot u_{2} \\
& =f_{2}(x)+g_{22} u_{2} \\
& \dot{x}_{3}=F_{31} \cdot x_{1}+g_{31}, u_{1}=f_{3}(x)+g_{31} u_{1} \\
& \dot{x}_{4}=F_{42} \cdot x_{2}+g_{42} \cdot u_{2}=f_{4}(x)+g_{42} u_{2} \\
& \dot{x}_{5}=F_{51} x_{1} x_{4}+F_{52} x_{2} \cdot x_{3}+F_{55} x_{5}+F_{56} \cdot x_{6}=f_{5}(x) \\
& \dot{x}_{6}=0=f_{6}(x) \\
& \text { Here, } F_{11}=F_{22}=\frac{-1}{\sigma}\left(\frac{R_{s}}{L_{s}}+\frac{1}{\tau_{r}}\right), F_{12}=-F_{21}=-N_{p} \\
& F_{13}=F_{24}=\frac{1}{\sigma . L_{s} \cdot \tau_{r}}, F_{14}=-F_{23}=\frac{N_{p}}{\sigma . L_{s}} \\
& F_{31}=F_{42}=-R_{s}, F_{51}=-F_{52}=\frac{-2 \cdot N_{p}}{3 . J_{m}}, F_{55}=\frac{-B_{m}}{J_{m}} \\
& F_{56}=-\frac{1}{J_{m}}, g_{11}=g_{22}=\frac{1}{\sigma \cdot L_{s}}, g_{31}=g_{42}=1 \\
& \sigma=1-\frac{M^{2}}{L_{s} \cdot L_{r}}, \tau_{r}=\frac{L_{r}}{R_{r}}
\end{aligned}
$$

Induction motor can thus be representated in a generalised form as in (7)

$$
\left.\begin{array}{l}
\dot{x}=f(x)+G . u \\
y=C \cdot x
\end{array}\right\}
$$

$G$ and $C$ are independent of states as follows,

$$
\begin{aligned}
G & =\left[\begin{array}{cccccc}
g 11 & 0 & g 31 & 0 & 0 & 0 \\
0 & g 22 & 0 & g^{42} & 0 & 0
\end{array}\right]^{T} \\
C & =\left[\begin{array}{llllll}
1 & 0 & 0 & 0 & 0 & 0 \\
0 & 1 & 0 & 0 & 0 & 0
\end{array}\right]
\end{aligned}
$$

The structure of the nonlinear observer proposed for SISO system in [6] and extended to a class of nonlinear MIMO systems by [7] is given by (8) and the schematic is shown in fig. 5 .

$$
\dot{\hat{x}}=f(\hat{x})+G(\hat{x}) \cdot u+Q^{-1}(\hat{x}, U) K[y-\hat{y}]
$$

here $K \in \Re^{(n \times m)}$ is the observer gain matrix and ' $U$ ' is the vector of inputs and their higher order derivatives. The main assumptions made in the design of observer are that the inputs are smooth and bounded, and the system nonlinearities are Lipschitz, atleast over the entire operating region. \{A vector function $F(x, t)$ is said to be locally Lipschitz in ' $x$ ' for a domain $\Omega \subset \mathfrak{R}^{\mathrm{n}}$ if, for any $\mathrm{x}_{1}, \mathrm{x}_{2} \in \Omega$ the following inequality holds: $\left\|F\left(x_{1}, t\right)-F\left(x_{2}, t\right)\right\| \leq a_{m}\left\|x_{1}-x_{2}\right\|, \forall t \in \Re$, where ' $a_{m}$ ' is a positive constant called Lipschitz constant of $F$ \}. The design makes use of a coordinate transformation $z=\phi(x, U)$, using Lie (directional) derivatives of $h(x)$ along $f(x)$. \{Lie derivative of ' $h(x)$ ' along ' $f(x)$ ' is defined as $\left.L_{f} h(x)=\frac{\partial h(x)}{\partial x} \cdot f(x)\right\}$. This transformation enables to introduce the concept of observability in the case of nonlinear systems. The observability matrix $Q$ $(x, U)$ is the Jacobian as given by (9). It usually depends on the states and higher order input derivatives, and should be nonsingular for reconstruction of state.

$$
Q(x, U)=\frac{\partial \phi(x, U)}{\partial x}
$$

After transformation, the system in new coordinates can be represented as in (10).

$$
\begin{aligned}
& \dot{z}=A z+B M(z, U) \\
& y=C z
\end{aligned}
$$

For applying to the induction motor, we select the coordinate trasformation as in (11).

$$
\begin{aligned}
& z=\phi(x, U)=\left[\begin{array}{llllll}
z_{11} & z_{12} & z_{13} & z_{21} & z_{22} & z_{23}
\end{array}\right]^{T} \\
& \text { where, } \\
& z_{11}=x_{1}=I_{s \alpha} \Rightarrow y_{1} \\
& z_{12}=\dot{x}_{1}=\dot{I}_{s \alpha} \\
& z_{13}=\ddot{x}_{1}=\ddot{I}_{s \alpha} \\
& z_{21}=x_{2}=I_{s \beta} \Rightarrow y_{2} \\
& z_{22}=\dot{x}_{2}=\dot{I}_{s \beta} \\
& z_{23}=\ddot{x}_{2}=\ddot{I}_{s \beta}
\end{aligned}
$$

Matrices $A, B$ and $C$, and the nonlinear function $M(z, U)$ are as in (12). 


$$
\begin{aligned}
A & =\left[\begin{array}{llllll}
0 & 1 & 0 & 0 & 0 & 0 \\
0 & 0 & 1 & 0 & 0 & 0 \\
0 & 0 & 0 & 0 & 0 & 0 \\
0 & 0 & 0 & 0 & 1 & 0 \\
0 & 0 & 0 & 0 & 0 & 1 \\
0 & 0 & 0 & 0 & 0 & 0
\end{array}\right], B=\left[\begin{array}{ll}
0 & 0 \\
0 & 0 \\
1 & 0 \\
0 & 0 \\
0 & 0 \\
0 & 1
\end{array}\right] \\
C & =\left[\begin{array}{llllll}
1 & 0 & 0 & 0 & 0 & 0 \\
0 & 0 & 0 & 1 & 0 & 0
\end{array}\right], M(z, U)=\left[\begin{array}{l}
M_{1}(z, U) \\
M_{2}(z, U)
\end{array}\right]
\end{aligned}
$$

It may be noted that, in the case of induction motor knowledge of higher order input derivatives is not necessary, as the observability matrix is independent of these derivatives. The assumption of boundedness of input is assured due to the physical limitation of inverter. These make the design simple

\section{Observer Stability}

The observer for the system in $\mathrm{z}$ - coordinate can be represented by (13). Corresponding dynamics of the state error $\left\{e_{z}=z-\hat{z}\right\}$ can be derived as in (14).

$$
\begin{aligned}
& \dot{\hat{z}}=A \hat{z}+B M(\hat{z}, U)+K[y-\hat{y}] \\
& \dot{e}_{z}=(A-K C) e_{z}+B[M(z, U)-M(\hat{z}, U)]
\end{aligned}
$$

State estimation in nonlinear systems becomes complicated because of the error dynamics being nonlinear. Hence, the convergence of the estimated state to true state cannot be guaranteed. To show the stability of the observer, a Lyapunov function $V=e_{z}{ }^{T} P^{-1} e_{z}$ is considered. The forward and inverse coordinate transformations are assumed to be Lipschitz continuous, and the nonlinear function $M(z, U)$ to be Lipschitz over the entire operating region. The condition for stability of the nonlinear observer is given by the $\mathrm{H}_{\infty}$ - Riccati equation (15).

$$
P(A-K C)^{T}+(A-K C) P+B B^{T}+\alpha P+a_{m}{ }^{2} P^{2} \leq 0
$$

where $P \in \Re(n \times n)$ is a symmetric positive definite matrix, $\alpha$ - observer convergance rate $>0, a_{m}$ - Lipschitz constant.

Once this condition is satisfied, the dynamics of the observer is as given by (16), which is proved in [7]

$$
\begin{aligned}
& \|x(t)-\hat{x}(t)\| \leq \mu . e^{-0.5 o t}\|x(0)-\hat{x}(0)\| \\
& \text { where, } \mu=\frac{\operatorname{Max}\{\operatorname{eig}(P)\}}{\operatorname{Min}\{\operatorname{eig}(P)\}}
\end{aligned}
$$

\section{Design}

The basic design steps for the nonlinear observer are summarized below.

1. Choose the Lipschitz constant ' $a_{m}$ ' associted with the nonlinear function $M(z, U)$ for a given operating region. Since it is difficult to determine a closed form relation between the function $\|M(z, U)\|$ and $\|z\|$, the Lipschitz constant associated with $M(z, U)$ is determined numerically. Thus, the Lipschitz constant for the transformed system is evaluated as in (17).

$$
\begin{aligned}
a_{m} & =\frac{\operatorname{Min}\{\|M(z, U)\|\}}{\operatorname{Max}\{\|z\|\}} \\
& \equiv \frac{\operatorname{Min}\{\|M(z, U)[2]-M(z, U)[1]\|\}}{\operatorname{Max}\{\|z[2]-z[1]\|\}}
\end{aligned}
$$

When the motor speed changes, $\|z\|$ varies. So ' $a_{m}$ ' also vary with speed as shown in fig. 6 and to cover the entire operating speed range, its maximum value is to be considered. Detailed procedure of evaluation of ' $a_{m}$ ' is given in Appendix - A.

2. Select the observer convergence rate ' $\alpha$ '. We select $\alpha$ equal to 0.6 .

3. Select the observer gain as $K=\lambda_{.} P C^{T}$, where $\lambda$ is a small positive constant and in this case set to 1. It may be noted that high values of ' $K$ ' will produce undesirable overshoot and oscillations.

4. Substitute the above relation in the the $\mathrm{H}_{\infty}$ - Riccati equation (14) and solve for ' $P$ '

5 . Use the relation in step 3 to obtain observer gain ' $K$ '.

\section{Simulation Results}

Simulation studies are done for the speed control of induction motor by Direct Torque Control method with nonlinear observer for the state estimation using the software tool MATLAB/SIMULINK ${ }^{\oplus}$. The nonlinear observer requires any two stator line currents and the inverter DC - link voltage as inputs for processing. Keeping in view of the typical high performance applications, performance specification's are set as; rise time equal to $0.4 \mathrm{sec}$, steady state speed error to be less than $1 \%$ and the damping to be critical. The inverter frequency is limited to $5 \mathrm{kHz}$. A reasonable delay of $1 \mathrm{~m}$ sec each is considered for the current and voltage sensors. Simulation results for a speed of $70 \mathrm{rad} / \mathrm{sec}$ is included. A load torque of $5 \mathrm{Nm}$ is applied to the motor at $1.5 \mathrm{sec}$ and released at $2.5 \mathrm{sec}$. Actual and estimated load torque characteristics are shown in fig. 7 . It can be seen that the estimate converges after $0.7 \mathrm{sec}$. This can be further lowered by an increase in the gain $K$, but results in an overshoot and oscillation, which is undesirable. Actual and estimated speed for $70 \mathrm{rad} / \mathrm{sec}$ with observer initial state errors set to $3 \mathrm{rad} / \mathrm{sec}$ for speed and 0.1 Weber for stator flux are shown in fig. 8 and fig. 9. The speed error and stator flux error convergence is shown in fig. 10 and fig. 11.

\section{Comments and Conclusions}

High-performance speed sensorless Direct Torque Control of induction motor under changing load conditions is the focus of this work. A nonlinear observer for the stator flux, speed, and load torque estimation is designed and developed for closed loop control operation. Simulation results reveal that the stator flux, speed and load torque regulations and their error convergence are guaranteed. The results are to be validated by implementing the algorithm in real time. This nonlinear observer requires the inverse calculation 
of the observability matrix, which increases the computational complexity. However, low cost and fast Digital Signal Processors capable of implementing relatively complex algorithms, are available in the market that makes this method suitable for high performance applications.

\section{Motor parameters}

$\mathrm{L}_{\mathrm{s}}=0.47 \mathrm{H}, \mathrm{L}_{\mathrm{r}}=0.47 \mathrm{H}, \mathrm{M}=0.44 \mathrm{H}, \mathrm{R}_{\mathrm{s}}=8 \Omega, \mathrm{R}_{\mathrm{r}}=3.6$ $\Omega, N_{\mathrm{p}}=2,\|U\|=180 \mathrm{~V},\left\|\mathrm{I}_{\mathrm{s}}\right\|=4.6 \mathrm{~A}, \varphi_{\mathrm{s} \text { [nom] }}=1.6 \mathrm{~Wb}$, $\mathrm{T}_{[\mathrm{nom}]}=7 \mathrm{~N}-\mathrm{m}, \omega_{\mathrm{t}[\mathrm{nom}]}=73 \mathrm{rad} / \mathrm{sec}, \mathrm{J}_{\mathrm{m}}=0.06 \mathrm{Kg}-\mathrm{m}^{2}, \mathrm{~B}_{\mathrm{m}}$ $=0.04 \mathrm{~N}-\mathrm{m}-\mathrm{sec}$.

\section{Figures}

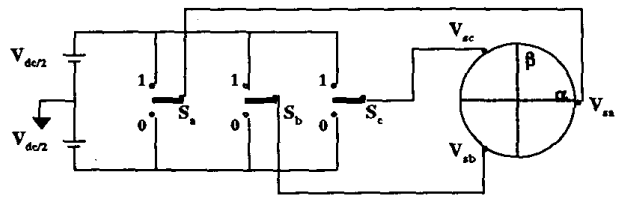

Fig. I Schematic Model of Inverter

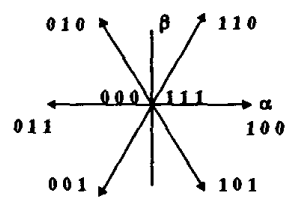

Fig. 2 Instantaneous Voltage Vectors

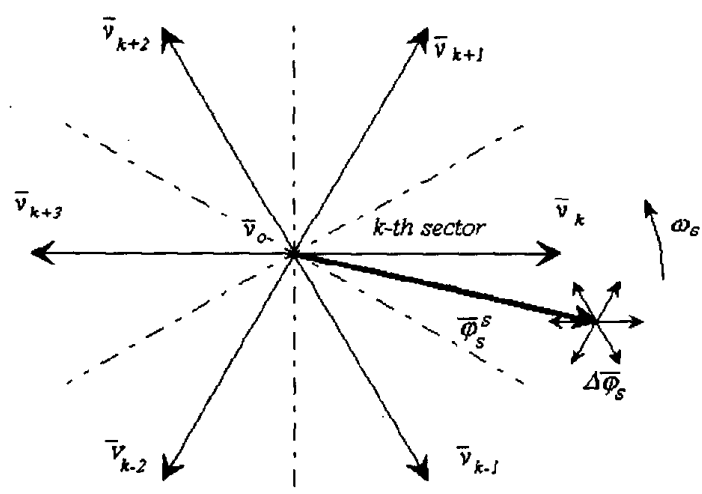

Fig. 3 Inverter Output Voltage Space Vectors and Corresponding Stator Flux Variations

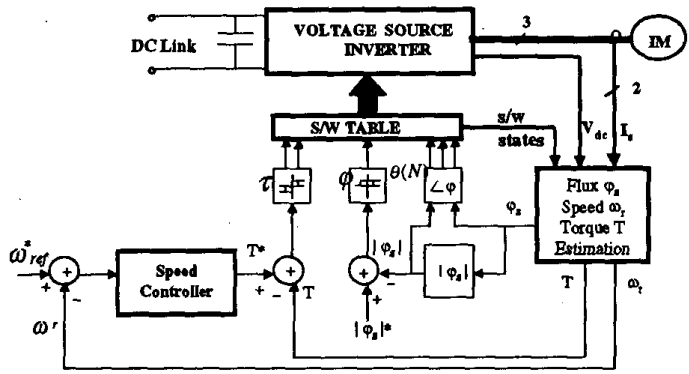

Fig.4 Schematic of DTC with Estimator

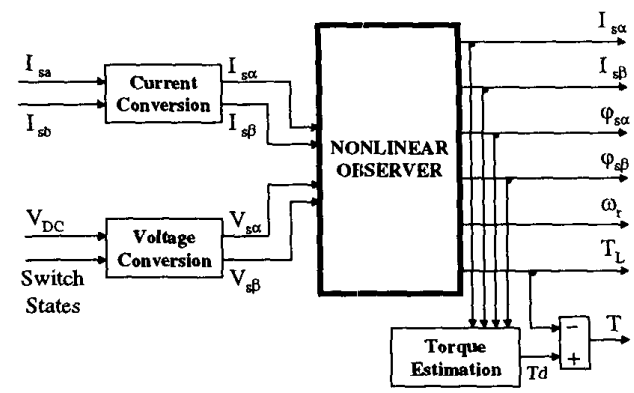

Fig. 5 Stator Flux, Speed, and $T_{L}$ Estimation

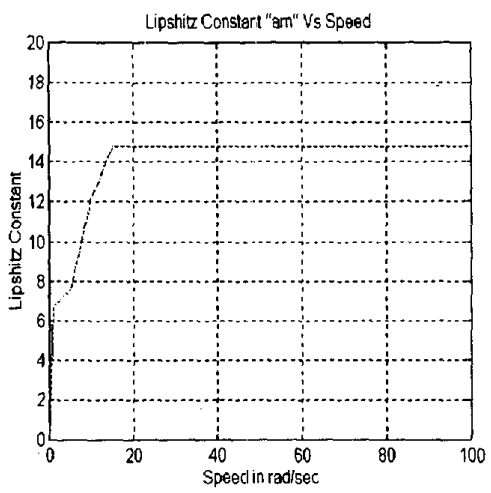

Fig. 6 Lipschitz constant ' $a_{m}$ ' Vs Speed curve

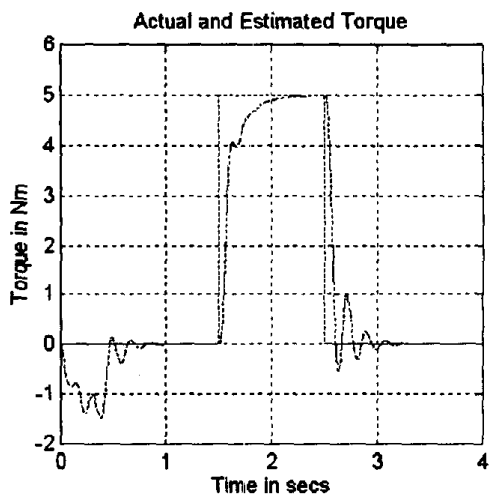

Fig. 7 Actual and Estimated $T_{L}$

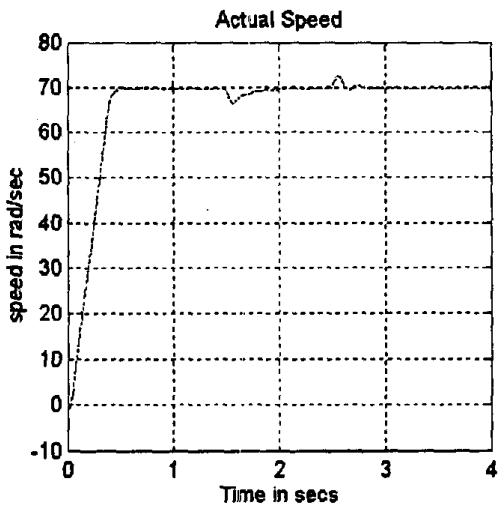

Fig. 8 Actual speed of motor 


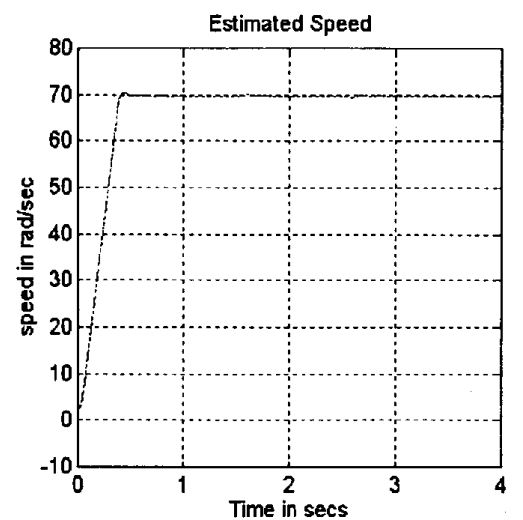

Fig. 9 Estimated speed of motor

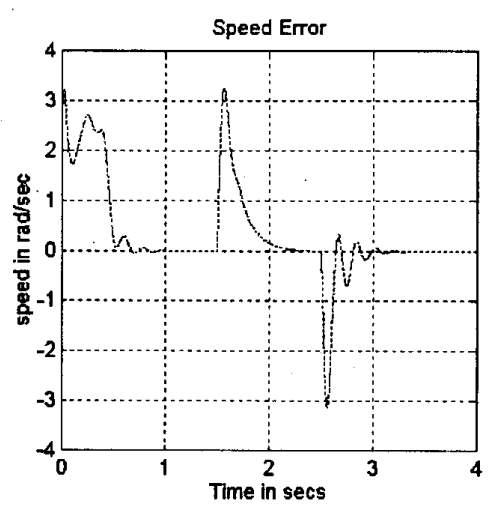

Fig. 10 Speed error convergance

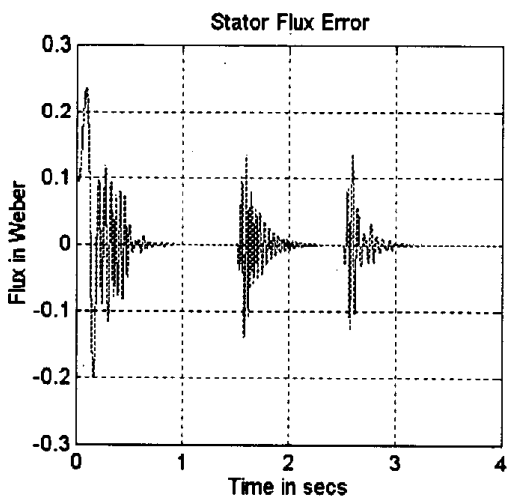

Fig. 11 Stator flux error convergance

\section{Appendix - A}

For the system in $z$-coordinate, it can be verified that $\left.M_{i}(z, U)\right|_{i=1,2}$ are the $3^{\text {rd }}$ and $6^{\text {th }}$ elements of $Q(x, U) . f(x)$. For calculating the Lipschitz constant for a particular speed, simulate the induction motor drive system without the observer for a reasonable time (include transient and steady state conditions) and obtain ' $k$ ' number of sampled datas each for $\left.z_{i}\right|_{i=1,2,3,4,5,6}$ and $\left.M_{i}(z, U)\right|_{i=1,2}$ where, $M(z, U)=\left[M_{1}(z, U) \quad M_{2}(z, U)\right]$ Find for, $i=1$ to 6 ,

$$
z_{i}[n]=z_{i}[j+1]-\left.z_{i}[j]\right|_{n=j=1,2, \ldots, k-1}
$$

and for, $i=1,2$

$$
M_{i}(z, U)[n]=M_{i}(z, U)[j+1]-\left.M_{i}(z, U)[j]\right|_{n=j=1,2, \ldots, k-1}
$$

Select the Lipschitz constant as the minimum of the slope given below and repeat the procedure for different speeds to obtain a plot as in fig. 6 .

$$
a_{m}=\operatorname{Min}\left\{a_{m}[n]\right\}=\operatorname{Min}\left\{\left.\frac{\left\|\left[M_{i}(z, U)[n]\right]\right\|}{\left\|\left[z_{i}[n]\right]\right\|}\right|_{n=1,2, \ldots, k-1}\right\}
$$

\section{References}

[1] Isao Takahashi, Toshibiko Noguchi, "A new quickresponse and high efficiency control strategy of an induction machine". IEEE Transactions on Industry Applications, vol. IA-22, no. 5, September/October 1986, pp. 820-827.

[2] James N Nash, "Direct -Torque Control, induction motor vector control without an encoder". IEEE Transactions on Industry Applications, vol. 33, March/April 1997, pp. 333-341.

[3] Pekka Tiitinen, M Surendra, "The next generation motor control method DTC, Direct Torque Control". Proceedings of EPE Chapter Symposium, Lausanne, Switzerland, 1994, pp. 37-43.

[4] Y A Chapuis, D Roye, J Davoine, "Principles and implementation of Direct Torque Control by stator flux orientation of an induction motor". Laboratoire d' Electro-technique de grenoble, E.N.S.I.E.G Domaine Universitaire, FRANCE.

[5] A M Walczyna, "Problems of application of Direct Flux and Torque Control methods to high power VSI-fed drives operating at low speed". Electrotechnical Institute, Department of Power Electronics, Warsaw, Poland.

[6] G Ciccarella, M Dalla Mora, A Germani, "A Luenberger like observer for nonlinear systems". International Journal of Control, vol. 57, no. 3 , 1993, pp. 537-556.

[7] Purnaprajna R Mangsuli, “Output Feedback Control of Nonlinear Systems with Application to Induction Motor". Ph.D. Thesis (submitted), Indian Institute of Science, Bangalore, 1999. 